Pathologe 2008 · [Suppl 2] 29:157-159

DOI 10.1007/s00292-008-1029-8

Online publiziert: 27. August 2008

(c) Springer Medizin Verlag 2008

F. Kommoss ${ }^{1}$. S. Kommoss ${ }^{2}$ - D. Schmidt ${ }^{1}$ A. Au Bois ${ }^{2}$. J. Pfisterer ${ }^{3}$

${ }^{1}$ Institut für Pathologie, Referenzzentrum für Gynäkopathologie, Mannheim

${ }^{2}$ Gynäkologie \& Gynäkologische Onkologie, Dr. Horst Schmidt Klinik (HSK), Wiesbaden

${ }^{3}$ Klinik für Gynäkologie und Geburtshilfe, Universitätsklinikum

Schleswig-Holstein, Campus Kiel

\title{
Histopathologische Zweitbegutachtung
}

\section{Einschlusskriterium für klinische Studien bei Ovarialkarzinomen?}

Abgrenzung epithelialer vs. nichtepithelialer Tumoren und in der Erkennung von Ovarialmetastasen [5]. Vergleichbare Ergebnisse zeigte eine Untersuchung aus England, bei der nach erneuter Begutachtung durch spezialisierte Gynäkopathologen in 27/64 (42\%) aller Fälle BOT des Ovars ursprünglich als Karzinom eingestuft worden waren [3]. Schließlich wurden 41/140 (29\%) Ovarialkarzinome nach histologischer Zweitbegutachtung als BOT reklassifiziert. Nur 4,5\% der Patientinnen mit korrigierter BOT-Diagnose verstarben an ihrer Krankheit, im Vergleich zu 25,6\% aller Patientinnen mit bestätigter Karzinomdiagnose [2].

Somit bleibt festzuhalten, dass ein gewisser Prozentsatz der Patientinnen in
Ovarialkarzinomstudien gemäßretrospektiver Analysen offensichtlich andere Läsionen aufweist, was im Widerspruch zu den Einschlusskriterien dieser Studien stehen kann. Es erscheint gut vorstellbar, dass für einige Studienpatientinnen mit Ovarialtumoren therapieassoziierte Morbidität ohne jeglichen oder mit nur geringem Benefit bei hohen Kosten entstehen könnte. Weiterhin könnten manchen Patientinnen andere/bessere Therapiemodalitäten vorenthalten werden. Es ist unser Ziel, diese Annahmen und damit die Sinnhaftigkeit einer spezialisierten histopathologischen Zweitbegutachtung bei Ovarialkarzinomen durch eine prospektive Untersuchung zu prüfen.

mäß den WHO-Kriterien [4] bestätigt. 19/319 (5,9\%) der Fälle entsprachen Borderline-Tumoren (BOT). Von den 19 Patientinnen mit ,sekundären BOT“ starben nur $3(15,7 \%)$ an ihrem Tumorleiden, im Vergleich zu 196/300 (65,3\%) der Patientinnen mit referenzpathologisch bestätigter Karzinomdiagnose.

In der Literatur finden sich weitere Hinweise auf signifikante Diskrepanzen zwischen Primärdiagnosen und Zweitbegutachtung bei Ovarialkarzinomen. Von 477 in den Jahren 1980 bis 1982 beschriebenen Ovarialkarzinomen aus einer Fallkontrollstudie wurden nach einer spezialisierten Zweitbegutachtung 57 (15\%) der Fälle als BOT reklassifiziert. Unterschiede zwischen dezentraler Pathologie und Expertenpanel ergaben sich zudem bei der

Abb. 1 Ablauf der histopathologischen Zweitbegutachtung im Verlauf der AGO-OVAR$11 \mathrm{t}$-HISTO-Studie

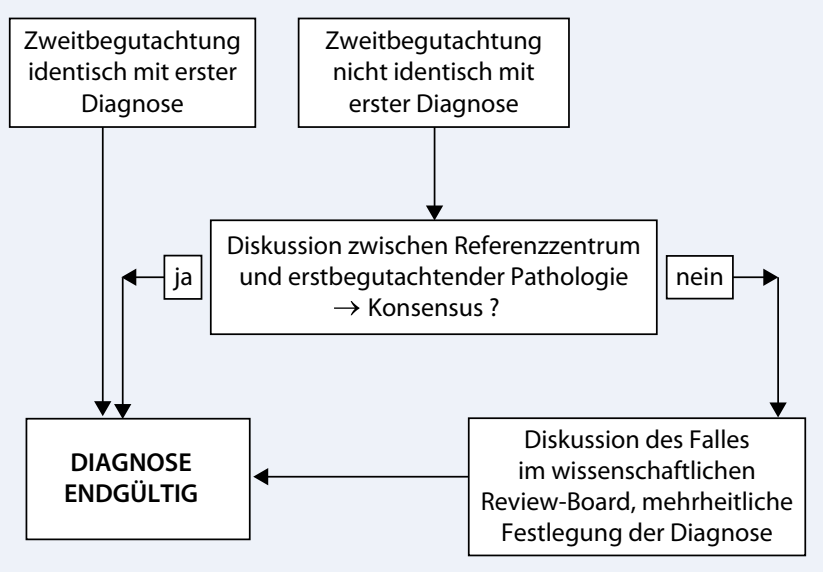


Pathologe 2008 • [Suppl 2] 29:157-159 DOI 10.1007/s00292-008-1029-8

(c) Springer Medizin Verlag 2008

\section{F. Kommoss · S. Kommoss · D. Schmidt · A. du Bois · J. Pfisterer \\ Histopathologische Zweitbegutachtung. Einschlusskriterium für klinische Studien bei Ovarialkarzinomen?}

\section{Zusammenfassung}

Eine histopathologische Zweitbegutachtung ist derzeit nicht Standard in klinischen Studien von Ovarialkarzinomen. Gemäß publizierter retrospektiver Analysen und eigener unveröffentlichter Erfahrungen hat ein gewisser Prozentsatz der Patientinnen in diesen Studien andere Läsionen, was im Widerspruch zu den Einschlusskriterien dieser Studien stehen kann. Als problematisch hat sich hierbei die Abgrenzung invasiver Ovarialkarzinome von Borderline-Tumoren, von Ovarialmetastasen sowie von Keimstrangtumoren erwiesen. Im Falle einer Fehldiagnose könnte einerseits therapieassoziierte Morbidität ohne Benefit bei dennoch hohen Kosten entstehen, andererseits könnten manchen Patientinnen die der eigentlichen Läsion besser ent- sprechenden Therapiemodalitäten vorenthalten werden. Die Sinnhaftigkeit einer zentralen histopathologischen Zweitbegutachtung als Einschlusskriterium für klinische Studien bei Ovarialkarzinomen wird derzeit prospektiv im Rahmen der AGO-Ovar-Studie „HIstologische STandardisierte Zweitbegutachtung in einem Studienkollektiv fortgeschrittener Ovarialkarzinome, OVAR 11t-HISTO" überprüft (http://www.mh-hannover.de/institute/ pathologie/dgp:Studien).

\section{Schlüsselwörter}

Ovarialkarzinom $\cdot$ Histopathologie $\cdot$ Zweitbegutachtung $\cdot$ Klinische Studien · Einschlusskriterium

\section{Central pathology review. Inclusion criterion for clinical studies of ovarian carcinomas?}

\begin{abstract}
Currently histopathological review is not standard practice in clinical studies of ovarian carcinomas. Published retrospective analyses as well as own unpublished observations have indicated that a certain percentage of study patients may have ovarian lesions other than ovarian carcinomas which may be in conflict with study inclusion criteria. In this context, the distinction of ovarian carcinomas from borderline tumors, ovarian metastases, and sex cord tumors has been shown to be a potential pitfall. In cases of incorrect diagnoses, non-beneficial morbidity at unnecessary high cost may occur, in other instances, more adequate therapeutic modalities might be withheld from a patient. At present, the
\end{abstract}

concept of a central histopathology review for patients with ovarian carcinomas is being studied prospectively in a translational research project of the AGO-Ovar termed "HIstologische STandardisierte Zweitbegutachtung in einem Studienkollektiv fortgeschrittener Ovarialkarzinome, OVAR 11t-HISTO" (Histological standardized review in a study collective of advanced ovarian cancer; http:// www.mh-hannover.de/institute/pathologie/ dgp:Studien).

\section{Keywords}

Ovarian carcinoma · Histopathology · Central pathology review . Clinical studies · Inclusion criterion

\section{Methoden und Resultate}

Unter der Schirmherrschaft der Studienleitgruppe der AGO-Ovar wurde die Studie „HIstologische STandardisierte Zweitbegutachtung in einem Studienkollektiv fortgeschrittener Ovarialkarzinome, OVAR 11t-HISTO“ initiiert. Hierbei handelt es sich um ein translationales Subprojekt der AGO-Ovar-11-Studie, einer derzeit offenen prospektiven internationalen multizentrischen Studie, welche die Bedeutung von Bevacicumab (Avastin $^{\circledR}$ ) im Zusammenhang mit dem derzeit etablierten Therapiestandard bei fortgeschrittenen Ovarialkarzinomen (Paclitaxel/Carboplatin-Chemotherapie nach radikaler operativer Tumorentfernung) untersucht.

Hauptfragestellung der OVAR-11t-HISTO-Studie ist es, die Hypothese: ",5\% der histologischen Primärdiagnosen Ovarialkarzinom sind in Studienkollektiven möglicherweise nicht zutreffend" zu überprüfen. Im Nebenschluss soll eine homogenisierte Tumorbank mit Paraffinmaterial der Ovarialkarzinome für die prospektive Prüfung gemäß vorläufiger Ergebnisse prognostisch und therapeutisch möglicherweise relevanter Faktoren etabliert werden.

Momentan gibt es 307 aktive klinische AGO-Ovar-Studienzentren. Diese arbeiten mit 206 pathologischen Instituten zusammen, darunter befinden sich 34 Universitätsinstitute, 96 Krankenhausabteilungen/Prosekturen und 76 Praxen. Aufklärung und Einverständniserklärung der Patientinnen für die OVAR-11t-HISTOStudie erfolgen in den klinischen Studienzentren unabhängig von der OVAR-11Studie auf separaten Formularen. Die Anmeldung zur OVAR-11t-HISTO-Studie erfolgt über das Internet durch die Kliniker, wobei 4 Informationen übermittelt werden:

1. eine Ovar-11-Identifikationsnummer,

2. die Histologienummer der erstbegutachtenden Pathologie,

3. die Diagnose, mit welcher die Patientin in die Studie eingebracht wurde,

4. das kooperierende pathologische Institut.

Das Studiensekretariat nimmt daraufhin Kontakt zur erstbegutachtenden Patho- 
logie auf, mit der Bitte um anonymisierte Übersendung sämtlicher Schnitte und Paraffinblöcke des betreffenden Falls zur Zweitbegutachtung im Institut für Pathologie, Referenzzentrum für Gynäkopathologie, A2/2 in Mannheim. Mit dieser Materialanforderung wird ein institutsspezifischer Zugangscode übermittelt, mit dessen Hilfe ein „log in“ auf der OVAR-11tHISTO-Homepage (http://www.ovarıthisto.de) möglich wird, auf der jederzeit der Stand der Zweitbegutachtung und der Verbleib des Materials abgefragt werden können. Zusätzlich werden ein Adresskleber für den Materialversand und ein Verrechnungsscheck über 25 EUR als Aufwandsentschädigung (gemäß der Empfehlungen des Berufsverbandes Pathologie) verschickt.

Nach Zweitbegutachtung der Fälle in Mannheim ist das weitere Vorgehen von deren Ergebnis abhängig (• Abb. 1). Stimmen Erstdiagnose und Zweitbegutachtung überein, werden sämtliche Schnitte und alle nicht zur Aufnahme in die AGO-Ovar-Tumorbank überlassenen Blöcke in die kooperierende Pathologie zurück versandt. Sind die Diagnosen nicht identisch, nimmt Mannheim Kontakt mit dem kooperierenden Institut auf, um die Befunde zu diskutieren und einen diagnostischen Konsensus zu erreichen. Findet eine Einigung statt, wird der Konsensus als studieninterne Diagnose verwendet. Im unwahrscheinlichen Falle einer bleibenden Diskrepanz werden die Präparate einem dreiköpfigen wissenschaftlichen „Review Board“ vorgelegt (Prof. Dr. J. Diebold, Luzern; Prof. Dr. S. Hauptmann, Halle; Prof. Dr. F. Kommoss, Mannheim). Das mehrheitliche Urteil dieses Expertenpanels wird dann als Studiendiagnose gewertet. Das detaillierte Studienprotokoll kann über die Webseiten der Deutschen Gesellschaft für Pathologie heruntergeladen werden [1].

International ist die AGO-Ovar-11-Studie für etwa 1500 Patientinnen offen. Von den ursprünglich etwa 500 für Deutschland vorgesehenen Patientinnen wurden bis zum Zeitpunkt der Zwischenauswertung (15.05.2008) bereits gut die Hälfte $(n=253)$ aufgenommen. Hiervon wurden 197/253 (77,8\%) bereits zusätzlich für OVAR-11t-HISTO rekrutiert. Bisher wurde Blockmaterial von 144 Fällen in einer
Tumorbank archiviert. Diskrepante Diagnosen wurden bereits beobachtet, über deren Art und genaue Zahl die abschließende Auswertung nach Ende der Patientenrekrutierung Aufschluss geben wird.

\section{Diskussion und Schlussfolgerung}

Histopathologische Zweitbegutachtung in Studien bei Ovarialkarzinomen könnte zumindest in Einzelfällen das Kollektiv durch Diskussion kontroverser Diagnosen homogenisieren und dadurch die wissenschaftliche Aussagekraft erhöhen. Sie könnte zudem Patientinnen der tumorspezifisch vorgesehenen Therapie zuführen oder nicht indizierte Therapien vermeiden helfen. Die Schaffung homogener Gewebebanken könnte die Initiierung prospektiver translationaler Untersuchungen wesentlich erleichtern. Neben der noch ausstehenden prospektiven Evidenz für die Sinnhaftigkeit einer histopathologischen Zweitbegutachtung bei Ovarialkarzinomen bestehen in diesem Zusammenhang jedoch noch ungelöste Probleme. Es ist bislang unklar, ob und inwieweit das derzeit mit Recht sehr kontrovers diskutierte Konzept referenzpathologischer Zweitbegutachtungen zukünftig gesellschaftlich und berufspolitisch erwünscht und weiterentwickelt werden soll.

Die Autoren vertreten die Ansicht, dass eine routinemäßige Zweitbegutachtung von Ovarialtumoren in aller Regel nicht erforderlich ist. Eine Zweitbegutachtung der Fälle in Studienkollektiven erscheint uns andererseits richtig und wichtig. Es müsste allerdings geklärt werden, wie die anfallende, nicht unerhebliche Mehrarbeit finanziert, sinnvoll verteilt und geleistet werden könnte. Hierfür müsste zunächst eine konstruktive Diskussion unter den am Thema interessierten Pathologen und Klinikern erfolgen mit dem Ziel einer Implementierung geeigneter Netzwerke. Um eine Zweitbegutachtung als Einschlusskriterium für klinische Studien sinnvoll in den therapeutischen Algorithmus zu integrieren, wäre eine konsequente Weiterentwicklung internetbasierter Netzwerke und Verteilungssysteme erforderlich. Mit dem Projekt OVAR 11t-HISTO werden wichtige Vorarbeiten geleistet, und die
Autoren wären gerne bereit, an der Weiterentwicklung mitzuarbeiten.

\section{Korrespondenzadresse}

Prof. Dr. F. Kommoss

Institut für Pathologie, Referenzzentrum für

Gynäkopathologie

A2/2, 68159 Mannheim

kommoss@gyn-patho.de

Interessenkonflikt. Der korrespondierende Autor weist auf folgenden Beziehungen hin: Die Studien AGO Ovar 11 und AGO Ovar 11t-HISTO werden finanziell gefördert durch MRC (Medical Research Council, London) und die Firma Roche.

\section{Literatur}

1. Deutsche Gesellschaft für Pathologie (2008) http:// www.mh-hannover.de/institute/pathologie/dgp: Studien

2. Leitao MM, Boyd J, Hummer A et al. (2004) Clinicopathologic analysis of early-stage sporadic ovarian carcinoma. Am J Surg Pathol 28: 147-159

3. Sengupta PS, Shanks JH, Buckley CH et al. (2000) Requirement for expert histopathological assessment of ovarian cancer and borderline tumours. $\mathrm{Br}$ J Cancer 82: 760-762

4. Tavassoli FA, Devilee P (2003) Pathology and genetics of tumours of the breast and female genital organs. International Agency for Research on Cancer, Lyon

5. Tyler CW, Lee NC, Robboy SJ et al. (1991) The diagnosis of ovarian cancer by pathologists: How often do diagnoses by contributing pathologists agree with a panel of gynecologic pathologists? Am J Obstet Gynecol 164: 65-70 